\title{
List of Reviewers
}

The following individuals have been kind enough to serve as ad hoc reviewers for Volumes 19, 20, 21 , and 22.

Diego Albani
Antonella Alberici
Dan Alkon
Alejandra Alonso
Annick Alperovitch
Alejandra Alvarez
Daniel Amen
David Ames
Gary Arendash
Jose Argimon Pallas
John Ashburner
Iris Asllani
Hani Atamna
Ali Atri
Johannes Attems
Craig Atwood
Brian Austen
Jesus Avila
Claudio Babiloni
Jan-Philipp Bach
Jason Bailey
Basavaraj Balapal
Emmanuel Barbeau
Deborah Barnes
Alfred Barth
David Bartrés-Faz
George Bartzokis
Ajoy Basak
James Becker
Andrei Belousov
David Bennett
Federico Bilotta
Giuliano Binetti
Garth Bissette
Alan Bittles
Alessandra Bizzarro
Eric Blalock
David Blum
Bradley Boeve
Detlev Boison

Arun Bokde
Istvan Boldogh
Alexander Boldyrev
Amy Borenstein
Barbara Borroni
Constantin Bouras
Gene Bowman
Adam Boxer
Andrea Bradford
Carol Brayne
John Breitner
Johannes Brettschneider
Gregory Brewer
Jean-Pierre Brion
Henry Brodaty
David Brown
David Bruce
Annador Bruce-Keller
Andrew Budson
Jeff Burns
Herman Buschke
Jorge Busciglio
Ashley Bush
D. Allan Butterfield
Frederic Calon
Carlo Caltagirone
Arezoo Cambell
Antoni Camins
Fabien Campagne
Dominique Campion
Giuseppina Candore
Jose Cantero
Chuandai Cao
Cristiano Capurso
Augusto Carlesimo
Anna Carnini
David Carpenter
Jose Carrion-Baralt
Gemma Casadesus
John Cashman

Jean Christophe Cassel
Angel Cedazo-Minguez
Balu Chakravarthy
Abba Chauhan
Cidi Chen
Hu Chen
Jiang-Fan Chen
Gael Chetelat
Dehua Chui
Jordi Clarimon
Carlo Colantuoni
Fabienne Collette
Joanna Collingwood
Carol Colton
Colin Combs
Cristoforo Comi
Annamaria Confaloni
Donald Connor
James Connor
David Cook
Jeff Coombes
Agata Copani
Elizabeth Corder
Carl Cotman
Eliecer Coto
James Cottrell
Elizabeth Coulson
Douglas Coursin
Bernard Croisile
Greg Crucian
Christian Czech
Balazs Czigler
Bruno da Silva
Jill Daniel
Jean-Francois Dartigues
Jack de la Torre
Bart De Strooper
Catherine Demko
Ilse Dewachter
David DeWitt

Jean Christophe Cassel

Balu Chakravarthy

Abba Chauhan

Cidi Chen

$\mathrm{Hu}$ Chen

Jiang-Fan Chen

Dehua Chui

Jordi Clarimon

Carlo Colantuoni

Fabienne Collette

Carol Colton

Colin Combs

Cristoforo Comi

Annamaria Confaloni

David Cook

Jeff Coombes

Agata Copani

Elizabeth Corder

Elizabeth Coulson

Douglas Coursin

Bernard Croisile

Balazs Czigler

Bruno da Silva

Jill Daniel

Dartigues

Catherine Demko

David DeWitt 
Emilio Di Maria

Brad Dickerson

Dennis Dickson

Eva Dierckx

James DiNardo

Jose Domingo

John Donahue

Fergus Doubal

Alexander Drzezga

Connie Duncan

Charles Duyckaerts

Barney Dwyer

Barbara Eckel

Gunter Eckert

David Eide

Gregory Elder

Heiner Ellgring

Carla Emiliani

Jacques Epelbaum

Jason Eriksen

Deniz Erten-Lyons

Herman Esselmann

Douglas Ewbank

Colette Fabrigoule

Anne Fagan

Gonzalo Farias

Franz Fazekas

Douglas Feinstein

Andreas Fellgiebel

John Feng

Joaquim Ferreira

Isidro Ferrer

Howard Fillit

Bonnie Firestein

Annette Fitzpatrick

Francoise Forette

Gianluigi Forloni

Blas Frangione

Chris Frederickson

Dan Frenkel

Avi Friedlich

Giovanni Frisoni

Jean-Pierre Fryns

Ansgar Furst

Andrea Fuso

Andrea Gaedigk

Douglas Galasko

Elena Galea

Daniela Galimberti

Hans-Joachim Galla

David Gallo
Jean-Marc Gallo

Francisco Garcia-Sierra

Brett Garner

James Geddes

Jonathan Geiger

Dwight German

Changiz Geula

Bernardino Ghetti

Giorgio Giaccone

Ezio Giacobini

Gary Gibson

Grover Gilmore

Anna Rita Giovagnoli

Ewa Golanska

Alexandra Golby

Harry Goldsmith

Carmen Gomez

Estrella Gómez-Tortosa

Cheng-xin Gong

Christian Gonzalez-Billault

Francisco Gonzalez-Lima

Jay Gottfried

Dawei Gou

Gunnar Gouras

IIlana Gozes

Caroline Graff

Ann-Charlotte Granholm

J. Timothy Greenamyre

David Greenberg

Joanna Groden

Francine Grodstein

Edna Grunblatt

Gilles Guillemi

Eugenia Gurevich

Annakaisa Haapasalo

Gerhard Hamann

Harald Hampel

Jing-Dong Han

John Hardy

Jarek Harezlak

Denise Harold

Charles Harrington

Alan Harvey

Jenni Harvey

Eli Hatchwell

Elizabeth Head

Felix Hernandez

Daniel Herrera

Helmut Hildebrandt

Hubertus Himmerich

John Hodges
Hyang-Sook Hoe

Barry Hoffer

Eef Hogervorst

Clive Holmes

Emily House

David Howlett

Chaur-Jong $\mathrm{Hu}$

Jacques Hugon

Christian Humpel

Christelle Hureau

Anja Hviid Simonsen

Emmanuel Ifeachor

Harry Ischiropoulos

Claudia Jacova

Helene Jacqmin

Chris Janus

Frank Jessen

Brian Jeynes

Jack Jhamandas

Conrad Johanson

John Johnson

Janet Johnston

Corinne Jolivalt

Joaquin Jordan Bueso

James Joseph

Carmen Junque

Elmar Kaiser

Jayne Kalmar

Ilyas Kamboh

Angela Kamer

David Kang

Anumantha Kanthasamy

Arlene Kasprisin

Dan Kaufer

John Kauwe

Jeffrey Kaye

Dirk Keene

Jeff Keller

Gerd Kempermann

Helene Kergoat

Amanda Kiliaan

Bhumsoo Kim

Chang-Ju Kim

Hideo Kimura

Becky Kinkead

Christopher Kipps

Alfredo Kirkwood

Eric Klann

Daniel Kopf

Hilary Koprowski

Amos Korczyn 
Joel Kramer

Inna Kruman

Benno Küsters

Bud Kukull

Alexander Kurz

Anna Ladogana

Debomoy Lahiri

Amanda LaMarre

Jean-Charles Lambert

Jessica Langbaum

Elmar Langenfeld

Diogo Lara

Anis Larbi

Asenath LaRue

Helen Lavretsky

Giampiero Leanza

Isabelle Leber

Dolores Ledesma

Daniel Lee

Hyoung-gon Lee

Hyun-Pil Lee

Joo-Yong Lee

Cynthia Lemere

Jason Lerch

Alan Lerner

Efrat Levy

Chris Link

Fei Liu

Gang Liu

Giancarlo Logroscino

Francisco Lopera

Carlo Lovati

Jie Lu

Burt Lynn

Fernando Maestú

Dorota Majeswka

German Malaga

Jarema Malicki

John Mamo

Facundo Manes

Hari Manev

Jan-Eric Mansson

Gabriella Marcon

Daniel Marson

Ana Martinez

Gianvito Martino

Aleksandra Maruszak

Eliezer Masliah

Colin Masters

Carlo Masullo

Hiroshi Matsuda
Francesco Mattace-Raso

Elaine McColl

Gawain McColl

Cherie McCracken

Hilary McMahon

Claudio Mello

Alexandre Mendonca

Claude Messier

Jacques Micheau

Bill Milgram

Alex Mitchell

Daniel Mograbi

Andreas Monsch

Alon Monsonego

Inhee Mook-Jung

Bernd Moosmann

Davide Moretti

Dave Morgan

John Morley

James Mortimer

Franz-Josef Mueller

Elliott Mufson

Gerd Multhaup

Gerald Munch

David Munoz

Ian Murray

Jill Murrell

Amanda Myers

Benedetta Nacmias

Hironobu Naiki

Anil Nair

Astrid Nehlig

Grazyna Niewiadomska

Janko Nikolich-Zugich

Flavio Nobili

Agneta Nordberg

Chris Norris

Akihiko Nunomura

Ester Oh

Barry Oken

John Olichney

Catarina Oliveira

Isaac Onyango

Carlos Opazo

Jean-Marc Orgogozo

Francesco Orzi

Cees Oudejans

Alessandro Padovani

Anders Paetau

Jorge Palop

Alexander Panov
Harish Pant

Johannes Pantel

Francesco Panza

Dominik Paquet

Anant Paradkar

In-Chul Park

Chris Parsons

Giulio Pasinetti

Gregory Pastores

Jodi Pawluski

Jin-Jing Pei

Dale Pellegrino

Botond Penke

Catherine Pennington

Giancarlo Pepeu

Daniela Perani

Claudia Pereira

Alessandro Peri

Grace Petot

Eric Petrie

Jay Pettegrew

Axel Petzold

Clyde Phelix

Aria Piccardi

Claus Pietrzik

Christian Pike

Jane Plailly

Emmanuel Planel

Giuseppe Plazzi

John Polich

Anton Porsteinsson

Hunt Potter

Domenico Pratico

Jonathan Prince

Alberto Pupi

Chengxuan Qiu

Jacob Raber

Gil Rabinovici

Balmiki Ray

Bill Rebeck

Hemachandra Reddy

Andreas Reif

Barry Reisberg

Anne Remes

Gavin Reynolds

Ian Reynolds

Florence Richard

Gerard Ridgway

Erich Ringelstein

Blaine Roberts

Guido Rodriguez 
Ekaterina Rogaeva

Jae-Kyu Roh

Jonathan Rohrer

Gerwin Roks

Gustavo Roman

Allan Roses

Egill Rostrup

Douglas Rowland

Donald Royall

Colin Royse

George Ruben

Alicia Rubio

Eckart Ruther

Joanne Ryan

Hoon Ryu

Alfredo Sadun

Angelo Sala

Ahmad Salehi

David Salmon

Eric Salmon

Cristina Sampaio

Fidel Santamaria

Ismael Santa-Maria

Cliff Saper

Aleister Saunders

Ann Saunders

Alexandru Saveanu

Gennaro Schettini

Frederick Schmitt

Jonathan Schott

Bernard Schreurs

Elisabeth Schrijvers

Johannes Schroeder

Ana Sebastiao

Fereshteh Sedaghat

Johann Sellner

Davide Seripa

Alessandro Serretti

Hari Sharma

Gerry Shaw

Jian-Gang Shen

Claire Shepherd

Jiong Shi

Jonghan Shin

Barbara Shukitt-Hale

Einar Sigurdsson

Andy Simmons

Frederico Simoes do Couto
Brij Singh

Joanna Siuda

Ingmar Skoog

David Small

A. David Smith

Andrew Smith

Hilkka Soininen

Beka Solomon

B.J. Song

Hermona Soreq

Gianfranco Spalletta

Dan Spielman

John Starr

Glenn Stebbins

Blossom Stephan

Anne Stephenson

Erik Stomrud

Alexis Stranahan

Daqiang Sun

Claire Surr

Dick Swaab

Carlton Taft

Akihiko Takashima

Atsushi Takeda

Elena Tamagno

Davide Tampellini

Eng-King Tan

Heikki Tanila

Giuseppina Tesco

Bruce Teter

Charlotte Teunissen

Dietmar Thal

Jessie Theuns

Gopal Thinakaran

David Trotti

Ian Trounce

Andrew Tsin

Christophe Tzourio

Francesc Valldeoriola

Christine van Broeckhoven

Fred van Leuven

John van Swieten

John Varghese

Ana Verdelho

Giuseppe Verdile

Barbara Vicini Chilovi

Cathy Vickers

Harry Vinters
Anand Viswanathan

Ivana Vodopivec

Guenter Vollmer

Christine von Arnim

Douglas Walker

Anders Wallin

Chunyu Wang

Dengshun Wang

Hui-xin Wang

Jian-Zhi Wang

Amber Watts

Ed Weeber

Jerzy Wegiel

Howard Weiner

Cheryl Wellington

Eric Westman

Jennifer Whitwell

Christian Wider

Donna Wilcock

Anders Wimo

Jaime Winderbaum Fernandez

Jacek Witkowski

Hartwig Wolburg

Henrike Wolf

Catherine Wolkow

Ben Wolozin

Jack Wong

Jong Woo

Huaxi Xu

Dunsheng Yang

Benjamin Yee

Geda Yonas

David Yousem

Huang Yu

Berran Yucesoy

Edward Zamrini

Gianluigi Zanusso

Eva Zerovnik

Henrik Zetterberg

Alex Zhang

Hong Zhang

Jie Zhang

Jing Zhang

Guojun Zhao

Haining Zhu

Earl Zimmerman

Berislav Zlokovic 